\title{
Robust Hierarchical Control for Uncertain Multivariable Hexarotor Systems
}

\author{
Wei Lin ${ }^{1}$ and Hao Liu' \\ ${ }^{1}$ State Key Laboratory of Mathematical Engineering and Advanced Computing, No. 62 Science Road, Zhengzhou 450002, China \\ ${ }^{2}$ School of Astronautics, Beihang University, Beijing 100191, China
}

Correspondence should be addressed to Hao Liu; liuhao13@buaa.edu.cn

Received 12 February 2015; Accepted 29 April 2015

Academic Editor: Hiroyuki Mino

Copyright (C) 2015 W. Lin and H. Liu. This is an open access article distributed under the Creative Commons Attribution License, which permits unrestricted use, distribution, and reproduction in any medium, provided the original work is properly cited.

\begin{abstract}
Multirotor helicopter attracts more attention due to its increased load capacity and being highly maneuverable. However, these helicopters are uncertain multivariable systems, which pose a challenge for their robust controller design. In this paper, a robust two-loop control scheme is proposed for a hexarotor system. The resulted controller consists of a nominal controller and a robust compensator. The robust compensators are added to restrain the influences of uncertainties such as nonlinear dynamics, coupling, parametric uncertainties, and external disturbances. It is proven that the tracking errors are ultimately bounded with specified boundaries by choosing the parameters of the robust compensators. Simulation results on the hexarotor demonstrate the effectiveness of the proposed control method.
\end{abstract}

\section{Introduction}

Unmanned aerial vehicles (UAV) have attracted great attention in scientific research area in the last two decades (see [1-5] to mention a few). These kinds of vehicles have been widely used to perform civil and military missions such as inspection, surveillance, exploration, search, and rescue. Unmanned rotorcrafts have some advantages over the fixwing aircrafts due to their special way of thrust lift generated. The helicopters can hover and take off and land vertically. Furthermore, unmanned helicopters can enter the building and implement indoor exploration tasks as well as inspection missions.

Multirotors have received much interest in the automatic control field because they can outperform the conventional helicopters in many aspects. Firstly, the fixed-pitch rotors are used and lift thrusts can be altered by the control of motor rotational speeds without a swashplate [6]. Secondly, multiple rotors can increase payload and the maneuverability of the helicopter [7]. Thirdly, the usage of the multiple rotors guarantees that each individual rotor is smaller than the equivalent main rotor of a conventional helicopter for a given airframe size [8]. Multiple smaller rotors can be enclosed within a protective frame and thus increased safety of operators. As a result of the strong points mentioned above, intensive efforts have been devoted to the tracking control problem of multirotor helicopters. Quadrotors have gained much attention in the automatic control field (see, e.g., [9-15]). In this paper, the robust motion control problem of hexarotor helicopters is investigated. Compared with quadrotors, although hexarotor helicopters may present a weight and energy consumption augmentation due to the extra motors, they can increase load capacities. Moreover, hexarotors are highly maneuverable in respect that at least four rotors can influence the dynamics of each direction. On the other hand, it results in a more fault-tolerant mechanical system for each hexarotor helicopter.

The controller design problem of a hexarotor system shares some merits with that of the quadrotor helicopter. Both kinds of helicopters can be considered as rigid bodies and thus one can apply Lagrange approach to obtain the dynamical model of the two kinds of helicopters. However, there also exist some differences between the two kinds of helicopters. The differences result from the aerodynamic forces and torques generated by the rotors. Because of two extra rotors, the torque produced in each channel is different from the quadrotors and thus affects the dynamical response 


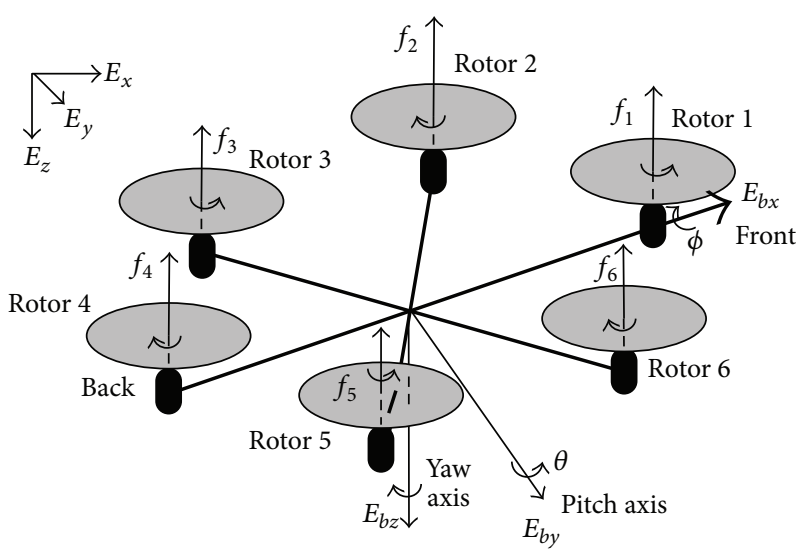

FIgURE 1: The schematic of the hexarotor helicopter.

differently. In this paper, a robust hierarchical control scheme is applied based on PD control method and the robust compensation approach. For each loop, a robust controller is designed with a nominal PD controller and a robust compensator. The robust compensators are introduced to restrain the influences of the uncertainties in both loops.

Compared to previous studies on the robust motion control problem of the multirotor helicopter, the proposed robust hierarchical control scheme can restrain disturbances in both the inner and outer loops. The tracking errors of this rotorcraft are guaranteed to be bounded with specified boundaries. In addition, this control scheme results in a linear time-invariant controller which is easily to be implemented in practical applications.

The remaining parts of this paper are organized as follows. In Section 2, the nonlinear mathematic model of the helicopter is described and problem description is presented. The nominal controllers and robust compensators in the attitude and position loops are designed in Section 3. In Section 4, the robust properties of closed-loop system by the designed control laws are proven. Simulation results are shown in Section 5 and conclusions are stated in Section 6.

For $y(t)=\left[y_{i}(t)\right] \in L_{\infty}^{n}$ and $h(t)=\left[h_{i j}(t)\right] \in L_{1}^{m \times n}$, denote that

$$
\begin{gathered}
\|y\|_{\infty}=\max _{i} \sup _{t \geq 0}\left|y_{i}(t)\right|, \\
\|H(p)\|_{1}=\|h\|_{1}=\max _{i}\left(\sum_{j} \int_{0}^{\infty}\left|h_{i j}(t)\right| d t\right),
\end{gathered}
$$

where $p$ is the Laplace operator, $H(p)=\ell(h(t))$, and $\ell(\cdot)$ indicates the Laplace transform.

\section{Problem Description}

The schematic of the hexarotor is depicted in Figure 1. Let $\alpha=$ $\left\{E_{x}, E_{y}, E_{z}\right\}$ denote an inertial frame and $\beta=\left\{E_{b x}, E_{b y}, E_{b z}\right\}$ is a frame attached to the helicopter body with origin in the center of the mass of the rotorcraft as shown in Figure 1. The vector $\xi=\left[\begin{array}{lll}x & y & z\end{array}\right]^{T}$ indicates the position of the origin of the body-fixed frame $\beta$ with respect to the inertial frame $\alpha$.
In addition, let $\eta=\left[\begin{array}{lll}\theta & \phi & \psi\end{array}\right]^{T}$ indicate the three Euler angles: the pitch angle $\theta$, the roll angle $\phi$, and the yaw angle $\psi$, which depends on the rotation from the inertial frame $\alpha$ to the bodyfixed frame $\beta$. The mathematical model of the helicopter can be derived by the Lagrangian method as (see, e.g., [9])

$$
\begin{aligned}
& m \ddot{\xi}=R f+m_{g e}, \\
& J \ddot{\eta}=-C(\eta, \dot{\eta}) \dot{\eta}+\tau,
\end{aligned}
$$

where $m$ is the mass of the helicopter, $J=\left[\begin{array}{lll}J_{\theta} & J_{\phi} & J_{\psi}\end{array}\right]$ is the inertia matrix of the helicopter relative to the frame $\alpha$, $g$ is the gravity constant, $C(\eta, \dot{\eta})$ is the Coriolis terms, $R=$ $\left[\begin{array}{lll}-\sin \theta & \cos \theta \sin \phi & \cos \theta \cos \phi\end{array}\right]^{T}, e_{3}=\left[\begin{array}{lll}0 & 0 & 1\end{array}\right]^{T}$, and $f$ and $\tau=\left[\begin{array}{lll}\tau_{\theta} & \tau_{\phi} & \tau_{\psi}\end{array}\right]^{T}$ are the external body-fixed frame force and torque respectively. $f$ and $\tau$ can be obtained as

$$
\begin{aligned}
& f=f_{1}+f_{2}+f_{3}+f_{4}+f_{5}+f_{6}, \\
& \tau_{\theta}=l_{r g}\left(f_{1}-f_{4}\right)+\frac{l_{r g}\left(f_{2}-f_{3}-f_{5}+f_{6}\right)}{2}, \\
& \tau_{\phi}=\frac{\sqrt{3} l_{r g}\left(f_{2}+f_{3}-f_{5}-f_{6}\right)}{2}, \\
& \tau_{\psi}=k_{f m}\left(f_{1}-f_{2}+f_{3}-f_{4}+f_{5}-f_{6}\right),
\end{aligned}
$$

where $l_{r g}$ is the distance from each motor to the center of the mass of the rotorcraft, $k_{f m}>0$ denotes the force-tomoment scaling factor, and $f_{i}(i=1,2, \ldots, 6)$ are the thrusts generated by the six rotors respectively. $f_{i}(i=1,2, \ldots, 6)$ take the following forms:

$$
f_{i}=k_{\omega} \omega_{i}^{2}, \quad i=1,2, \ldots, 6
$$

where $k_{\omega}$ is a positive constant and $\omega_{i}(i=1,2, \ldots, 6)$ are the rotational speeds of the six rotors respectively. Define the control inputs $u_{i}(i=1,2, \ldots, 4)$ as

$$
\begin{aligned}
& u_{1}=\omega_{1}^{2}+\omega_{2}^{2}+\omega_{3}^{2}+\omega_{4}^{2}+\omega_{5}^{2}+\omega_{6}^{2}, \\
& u_{2}=\omega_{1}^{2}-\omega_{4}^{2}+\frac{\left(\omega_{2}^{2}-\omega_{3}^{2}-\omega_{5}^{2}+\omega_{6}^{2}\right)}{2}, \\
& u_{3}=\frac{\sqrt{3}\left(\omega_{2}^{2}+\omega_{3}^{2}-\omega_{5}^{2}-\omega_{6}^{2}\right)}{2}, \\
& u_{4}=\omega_{1}^{2}-\omega_{2}^{2}+\omega_{3}^{2}-\omega_{4}^{2}+\omega_{5}^{2}-\omega_{6}^{2} .
\end{aligned}
$$

In practical applications, there already exist many power distribution boards to distribute the four control inputs to the six rotors for the multirotors. Therefore, the power distribution problem is not further discussed here. 
In addition, let

$$
\begin{aligned}
& b_{1}^{N}=-\frac{k_{\omega}^{N} u_{10}}{m^{N}}, \\
& b_{2}^{N}=\frac{k_{\omega}^{N} u_{10}}{m^{N}}, \\
& b_{3}^{N}=\frac{k_{\omega}^{N}}{m^{N}}, \\
& b_{4}^{N}=\frac{l_{r g}^{N} k_{\omega}^{N}}{J_{\theta}^{N}}, \\
& b_{5}^{N}=\frac{l_{r g}^{N} k_{\omega}^{N}}{J_{\varphi}^{N}}, \\
& b_{6}^{N}=\frac{k_{f m}^{N} k_{\omega}^{N}}{J_{\psi}^{N}},
\end{aligned}
$$

where the superscript $N$ denotes the nominal value of the helicopter parameter and $u_{10}$ is the value of control input $u_{1}$ in the hovering working point which is a positive constant. Then from (2), one can obtain that

$$
\begin{aligned}
& \ddot{x}=b_{1}^{N}\left(\theta+q_{1}\right), \\
& \ddot{y}=b_{2}^{N}\left(\phi+q_{2}\right), \\
& \ddot{z}=b_{3}^{N}\left(u_{1}+q_{3}\right)+g, \\
& \ddot{\theta}=b_{4}^{N}\left(u_{2}+q_{4}\right), \\
& \ddot{\phi}=b_{5}^{N}\left(u_{3}+q_{5}\right), \\
& \ddot{\psi}=b_{6}^{N}\left(u_{4}+q_{6}\right),
\end{aligned}
$$

where $q_{i}(i=1,2, \ldots, 6)$ are called equivalent disturbances and take the forms

$$
\begin{aligned}
& q_{1}=\frac{\left(-\sin \theta k_{\omega} u_{1} / m-b_{1}^{N} \theta\right)}{b_{1}^{N}}+d_{1}, \\
& q_{2}=\frac{\left(\cos \theta \sin \phi k_{\omega} u_{1} / m-b_{2}^{N} \phi\right)}{b_{2}^{N}}+d_{2}, \\
& q_{3}=\frac{\left(\cos \theta \cos \phi k_{\omega} / m-b_{3}^{N}\right) u_{1}}{b_{3}^{N}}+\frac{\left(m-m^{N}\right) g}{b_{3}^{N}}+d_{3}, \\
& q_{i}=\frac{\left(b_{i}-b_{i}^{N}\right) u_{i-2}}{b_{i}^{N}}+c_{i}+d_{i}, \quad i=4,5,6,
\end{aligned}
$$

where $d_{i}(i=1,2, \ldots, 6)$ are external disturbances and $c_{i}(i=$ $1,2,3)$ are higher order terms that result from the Coriolis terms.

Assumption 1. The pitch and roll angles are bounded among $(-\pi / 2, \pi / 2)$ and satisfy that $\theta \in\left[-\pi / 2+\delta_{\theta}, \pi / 2-\delta_{\theta}\right]$ and $\phi \in\left[-\pi / 2+\delta_{\phi}, \pi / 2-\delta_{\phi}\right]$ with $\delta_{\theta}$ and $\delta_{\phi}$ positive constants.
In order to avoid singularities in the Euler angle representation, the hexarotor is required to avoid overturning during the flight [16].

Assumption 2. The control input $u_{1}$ is bounded and satisfies that $u_{1} \in\left[\delta_{u 1}, 2 u_{10}-\delta_{u 1}\right]$, where $\delta_{u 1}$ is a positive constant.

Assumption 3. The uncertain parameters $b_{i}(i=4,5,6), k_{\omega}$, and $m$ are bounded. The nominal parameters $b_{1}^{N}$ are negative and $b_{i}^{N}(i=2,3, \ldots, 6)$ are positive and satisfy that $b_{1}^{N} \leq$ $-2 k_{\omega} u_{10} / \pi / m, b_{2}^{N} \geq 2 k_{\omega} u_{10} / \pi / m$, and $\left|b_{i}-b_{i}^{N}\right| \leq b_{i}^{N}(i=$ $3,4,5,6)$.

One can set that $-b_{1}^{N}$ are $b_{i}^{N}(i=2, \ldots, 6)$ with larger positive values, if $b_{i}^{N}(i=1,2, \ldots, 6)$ do not satisfy the above the assumption.

Define $\rho_{1}=-\left|k_{\omega} \sin \theta u_{1} / \theta / m+b_{1}^{N}\right| / b_{1}^{N}, \rho_{2}=$ $\left|k_{\omega} \cos \theta \sin \phi u_{1} / \phi / m-b_{2}^{N}\right| / b_{2}^{N}, \rho_{3}=\left|k_{\omega} \cos \theta \cos \phi / m+b_{3}^{N}\right| / b_{3}^{N}$, and $\rho_{i}=\left|b_{i}-b_{i}^{N}\right| / b_{i}^{N}(i=4,5,6)$.

If Assumptions 1, 2, and 3 hold, one can obtain that $0 \leq$ $\rho_{i}<1(i=1, \ldots, 6)$.

Assumption 4. The external disturbances $d_{i}(i=1, \ldots, 6)$ are bounded.

In the position control mode discussed here, the three positions and the yaw angle $\{x, y, z, \psi\}$ are chosen as outputs. This paper will investigate the robust controller design problem to achieve practical tracking of the prescribed reference signals $r_{i}(i=x, y, z, \psi)$ for $x, y, z$, and $\psi$, respectively.

Assumption 5. The reference signals $r_{i}(i=x, y, z, \psi)$ and their derivatives $\dot{r}_{i}(i=x, y, z, \psi)$ are piecewise uniformly bounded.

\section{Robust PD Controller Design}

Based on the PD control approach and the signal compensation method [17-21], the attitude and position controllers are designed in two steps.

From the helicopter system (7), one can see that the robust tracking control of the longitude and lateral directions can be achieved by controlling the attitude angles $\theta$ and $\varphi$ appropriately, if the three positions and the yaw angle are chosen as the outputs; that is, the position controller involved $x$ and $y$ positions that will be designed to produce the desired attitude signals $r_{\theta}$ and $r_{\varphi}$ for $\theta$ and $\varphi$ to track, whereas the vertical direction ( $z$-axis) can be dealt with independently. The attitude controller will be applied to track the references signals $r_{\theta}, r_{\varphi}$, and $r_{\psi}$ for the three attitude channels.

3.1. Position Controller Design. Define $u_{\xi 1}=\theta_{d}, u_{\xi 2}=\varphi_{d}$, $u_{\xi 3}=u_{1}+g / b_{1}^{N}$, and $e_{\xi i}=\left[\begin{array}{ll}e_{\xi i 1} & e_{\xi i 2}\end{array}\right]^{T}(i=1,2,3)$, where $e_{\xi 11}=x-r_{x}, e_{\xi 12}=\dot{e}_{\xi 11}, e_{\xi 21}=y-r_{y}, e_{\xi 22}=\dot{e}_{\xi 21}, e_{\xi 31}=$ $z-r_{z}$, and $e_{\xi 32}=\dot{e}_{\xi 31}$. The position motion equations of the rotorcraft can be expressed as space-state forms:

$$
\dot{e}_{\xi i}=A_{\xi_{i}} e_{\xi i}+B_{\xi_{i}}\left(u_{\xi_{i}}+q_{i}\right), \quad i=1,2,3,
$$


where

$$
\begin{aligned}
A_{\xi i} & =\left[\begin{array}{ll}
0 & 1 \\
0 & 0
\end{array}\right], \\
B_{\xi i} & =\left[\begin{array}{c}
0 \\
b_{i}^{N}
\end{array}\right] .
\end{aligned}
$$

The control inputs $u_{\xi i}(i=1,2,3)$ consist of two parts: the $\mathrm{PD}$ control input $u_{\xi i}^{\mathrm{PD}}$ and the robust compensating input $u_{\xi i}^{\mathrm{RC}}$; that is, the control input can be expressed as

$$
u_{\xi i}=u_{\xi i}^{\mathrm{PD}}+u_{\xi i}^{\mathrm{RC}}
$$

Firstly, PD feedback control laws are constructed as

$$
u_{\xi i}^{\mathrm{PD}}=-K_{\xi i} e_{\xi i}, \quad i=1,2,3,
$$

where $K_{\xi i}=\left[\begin{array}{ll}k_{\xi i}^{p} & k_{\xi i}^{d}\end{array}\right]$ is selected such that $A_{\xi i}-B_{\xi i} K_{\xi i}$ is Hurwitz.

Then, the robust compensation technique is used to restrain the influences of the equivalent disturbances $q_{i}(i=$ $1,2,3)$. The robust compensating inputs for the three channels can be obtained by the robust filters $F_{\xi i}(p)(i=1,2,3)$ as

$$
u_{\xi i}^{\mathrm{RC}}(p)=-F_{\xi i}(p) q_{i}(p), \quad i=1,2,3,
$$

where $F_{\xi_{i}}(p)=f_{\xi i} g_{\xi i} /\left(p+f_{\xi_{i}}\right) /\left(p+g_{\xi i}\right)$. From (9)-(13), the robust compensating inputs $u_{\xi i}^{\mathrm{RC}}(i=1,2,3)$ can be realized by

$$
\begin{aligned}
& \dot{z}_{\xi i 1}=-g_{\xi i} z_{\xi i 1}-g_{\xi i}^{2} e_{\xi i}+b_{i}^{N} u_{\xi i}, \\
& \dot{z}_{\xi i 2}=-f_{\xi i} z_{\xi i 2}+\left(f_{\xi i}+g_{\xi i}\right) e_{\xi i}+z_{\xi i 1}, \\
& u_{\xi i}^{\mathrm{RC}}=\frac{f_{\xi i} g_{\xi i}\left(z_{\xi i 2}-e_{\xi i}\right)}{b_{i}^{N}}, \quad i=1,2,3 .
\end{aligned}
$$

3.2. Attitude Controller Design. Define $u_{\eta}=\left[\begin{array}{lll}u_{2} & u_{3} & u_{4}\end{array}\right]$, $q_{\eta}=\left[\begin{array}{lll}q_{4} & q_{5} & q_{6}\end{array}\right]$, and $e_{\eta}=\left[e_{\eta i}\right]_{6 \times 1}$, where $e_{\eta 1}=\theta-r_{\theta}$, $e_{\eta 2}=\dot{e}_{\eta 1}, e_{\eta 3}=\varphi-r_{\varphi}, e_{\eta 4}=\dot{e}_{\eta 3}, e_{\eta 5}=\psi-r_{\psi}$, and $e_{\eta 6}=\dot{e}_{\eta 5}$. The position motion equations of the rotorcraft can be expressed as space-state forms:

$$
\dot{e}_{\eta}=A_{\eta} e_{\eta}+B_{\eta}\left(u_{\eta}+q_{\eta}\right)
$$

where

$$
A_{\eta}=\left[\begin{array}{llllll}
0 & 1 & 0 & 0 & 0 & 0 \\
0 & 0 & 0 & 0 & 0 & 0 \\
0 & 0 & 0 & 1 & 0 & 0 \\
0 & 0 & 0 & 0 & 0 & 0 \\
0 & 0 & 0 & 0 & 0 & 1 \\
0 & 0 & 0 & 0 & 0 & 0
\end{array}\right],
$$

$$
B_{\eta}=\left[\begin{array}{ccc}
0 & 0 & 0 \\
b_{4}^{N} & 0 & 0 \\
0 & 0 & 0 \\
0 & b_{5}^{N} & 0 \\
0 & 0 & 0 \\
0 & 0 & b_{6}^{N}
\end{array}\right] .
$$

Similar to the position controller design, the attitude control input $u_{\eta}$ is designed with two parts: the PD control input $u_{\eta}^{\mathrm{PD}}$ and the robust compensating input $u_{\eta}^{\mathrm{RC}}$, as follows:

$$
u_{\eta}=u_{\eta}^{\mathrm{PD}}+u_{\eta}^{\mathrm{RC}}=-K_{\eta} e_{\eta}-F_{\eta}(p) q_{\eta}(p),
$$

where $K_{\eta}=\left[\begin{array}{lll}K_{\eta 1} & K_{\eta 2} & K_{\eta 3}\end{array}\right], F_{\eta}(p)=\operatorname{diag}\left(F_{\eta 1}(p), F_{\eta 2}(p)\right.$, $\left.F_{\eta 3}(p)\right), K_{\eta i}=\left[\begin{array}{ll}k_{\eta i}^{p} & k_{\eta i}^{d}\end{array}\right], F_{\eta i}(p)=f_{\eta i} g_{\eta i} /\left(p^{2}+f_{\eta i} p+g_{\eta i} p+\right.$ $\left.f_{\eta i} g_{\eta i}\right)(i=1,2,3) . K_{\eta}$ is selected such that $A_{\eta}-B_{\eta} K_{\eta}$ is Hurwitz. The robust compensating input $u_{\eta}^{\mathrm{RC}}$ can be realized in a similar way as (14).

\section{Robust Properties Analysis}

In this section, the robust performances of the closed-loop system constructed as in the previous section will be analyzed in two steps. Firstly, it will be proven that the state of the vertical channel is bounded and the tracking error is bounded and will converge to any given neighborhood of the origin under the influences of the dynamics of other channels. Then, the proof of the robust properties of the other channels will be presented based on the analysis of the robust performances of the vertical channel.

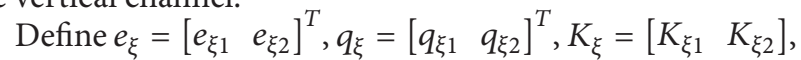
and $A_{\xi_{H}}=\operatorname{diag}\left\{A_{\xi_{1 H}}, A_{\xi_{2 H}}\right\}$. Combing (9)-(13), (15), and (17), one has

$$
\begin{aligned}
& \max _{j}\left|e_{i j}(t)\right| \leq \max _{j}\left|c_{i j}^{T} e^{A_{i H} t} e_{i}(0)\right|+\mu_{i}\left\|q_{i}\right\|_{\infty}, \\
& i=\xi, \xi 3, \eta,
\end{aligned}
$$

where $\mu_{i}=\left\|\left(p I_{i}-A_{i H}\right)^{-1} B_{i}\left(1-F_{i}\right)\right\|_{1}$ and $I_{i}$ is corresponding unit matrix. It follows that

$$
\left\|e_{i}\right\|_{\infty} \leq \gamma_{e_{i}(0)}+\mu_{i}\left\|q_{i}\right\|_{\infty}, \quad i=\xi, \xi 3, \eta,
$$

where $\gamma_{e_{i}(0)}=\max _{j} \sup _{t \geq 0}\left|c_{i j}^{T} e^{A_{i H} t} e_{i}(0)\right|$, and $c_{\xi j}, c_{\xi 3 j}$, and $c_{\eta j}$ are $4 \times 1,2 \times 1$, and $6 \times 1$ vectors with ones on the $j$ th row and 
zeros elsewhere, respectively. Define $g_{\xi}=\min \left\{g_{\xi 1}, g_{\xi 2}\right\}$ and $g_{\eta}=\min \left\{g_{\eta 1}, g_{\eta 2}, g_{\eta 3}\right\}$. If $f_{i}$ and $g_{i}(i=\xi 1, \xi 2, \xi 3, \eta 1, \eta 2, \eta 3)$ are sufficiently large and satisfy $f_{i} \gg g_{i}>0$, from [19], there exist positive constants $\gamma_{g i}(i=\xi, \xi 3, \eta)$ such that

$$
\mu_{i} \leq \frac{\gamma_{g i}}{g_{i}}, \quad i=\xi, \xi 3, \eta
$$

\subsection{The Robust Properties of the Vertical Channel}

Theorem 6. If Assumptions 1, 2, 3, and 5 hold, the closed-loop system of the vertical channel has robust tracking performances; that is, for any given positive constant $\varepsilon_{\xi 3}$ and any given bounded initial state, one can find positive constants $f_{\xi 3}, g_{\xi 3}$ with sufficiently large values and satisfying $f_{\xi 3} \gg g_{\xi 3}$, and $T_{\xi 3}$, such that, the state $e_{\xi 3}$ is bounded, and

$$
\left|e_{\xi 3}(t)\right| \leq \varepsilon_{\xi 3}, \quad \forall t \geq T_{\xi 3} .
$$

Proof. Under the Assumptions 1, 3, and 4, one has that

$$
\left\|q_{3}\right\|_{\infty} \leq \rho_{3}\left\|u_{\xi 3}\right\|_{\infty}+\left\|d_{3}\right\|_{\infty} .
$$

From (11)-(13) and (22), one can obtain two positive constants $\gamma_{e \xi 3}$ and $\gamma_{c \xi_{3}}$ such that

$$
\left\|q_{3}\right\|_{\infty} \leq \gamma_{e \xi 3}\left\|e_{\xi 3}\right\|_{\infty}+\gamma_{c \xi 3} .
$$

Then, from (19), (20), and (23), there exist positive constants $\varsigma_{q 3}$ and $\varsigma_{e \xi 3}$ satisfying

$$
\begin{gathered}
\left\|q_{3}\right\|_{\infty} \leq \gamma_{e_{\xi 3}(0)} \gamma_{e \xi 3}+\frac{\gamma_{g \xi 3} \gamma_{e \xi 3}\left\|q_{\xi 3}\right\|_{\infty}}{g_{\xi 3}}+\gamma_{c \xi 3} \leq \varsigma_{q 3}, \\
\left\|e_{\xi 3}\right\|_{\infty} \leq \gamma_{e_{\xi 3}(0)}+\frac{\gamma_{g \xi 3}\left\|q_{\xi 3}\right\|_{\infty}}{g_{\xi 3}} \leq \varsigma_{e \xi 3} .
\end{gathered}
$$

Furthermore, from (18), (20), and (24), one has

$$
\max _{j}\left|e_{\xi 3 j}(t)\right| \leq \max _{j}\left|c_{\xi 3 j}^{T} e^{A_{\xi_{3 H}} t} e_{\xi 3}(0)\right|+\frac{\gamma_{g \xi 3} \varsigma_{q 3}}{g_{\xi 3}} .
$$

Therefore, for any given positive constant $\varepsilon_{\xi 3}$, there exists a positive constant $T_{\xi 3}$ satisfying (21).

One can see that the dynamics of other channels cannot influence the robustness property analysis of the vertical channel and, thus, the values of $f_{\xi 3}$ and $g_{\xi 3}$ can be determined before discussing the tracking performances of other channels.

Lemma 7. There exist a positive constant $\varsigma_{u 10}$ such that the control input $u_{1}$ satisfies

$$
\left\|u_{1}\right\|_{\infty} \leq \varsigma_{u 10} .
$$

Proof. From (11)-(13) and (24), one can obtain a positive constant $\varsigma_{u 10}$ such that

$$
\begin{aligned}
\left\|u_{1}\right\|_{\infty} & \leq\left\|K_{\xi 3}\right\|_{1}\left\|e_{\xi 3}\right\|_{\infty}+\left\|q_{3}\right\|_{\infty}+\frac{\left\|\left(g-\ddot{r}_{z}\right)\right\|_{\infty}}{b_{3}^{N}} \\
& \leq \varsigma_{u 10} .
\end{aligned}
$$

Lemma 7 follows.
Theorem 8. If Assumptions 1 to 5 hold, the closed-loop system of the longitudinal, latitude, and three attitude channels have robust tracking performances; that is, for any given positive constants $\varepsilon_{\xi}$ and $\varepsilon_{\eta}$ and any given bounded initial state, one can obtain positive constants $i_{\xi 1}, i_{\xi 2}, i_{\eta 1}, i_{\eta 2}$, and $i_{\eta 3}(i=f, g)$ with sufficiently large values and satisfying $f_{i} \gg g_{i}(i=$ $\xi 1, \xi 2, \eta 1, \eta 2, \eta 3)$, and $T_{\xi}$ and $T_{\eta}$ such that, the states $e_{\xi}$ and $e_{\eta}$ are bounded, and

$$
\begin{aligned}
& \max _{i}\left|e_{\xi i}(t)\right| \leq \varepsilon_{\xi}, \quad \forall t \geq T_{\xi}, \\
& \max _{i}\left|e_{\eta i}(t)\right| \leq \varepsilon_{\eta}, \quad \forall t \geq T_{\eta} .
\end{aligned}
$$

Proof. From (8) and Lemma 7, one can obtain positive constants $\varsigma_{q \xi e \eta}$, $\varsigma_{q \xi \xi \xi}$, and $\varsigma_{q \xi c}$ such that

$$
\left\|q_{\xi}\right\|_{\infty} \leq \varsigma_{q \xi e \eta}\left\|e_{\eta}\right\|_{\infty}+\varsigma_{q \xi \xi \xi}\left\|e_{\xi}\right\|_{\infty}+\varsigma_{q \xi c} .
$$

From (8), (11), (12), (13), (17), (29), and Lemma 7, there exist positive constants $\varsigma_{q \eta e \eta}, \varsigma_{q \eta e \xi}, \varsigma_{q \eta e \eta 2}, \varsigma_{q \eta e \xi 2}, \varsigma_{q \eta e \eta \xi}$, and $\varsigma_{q \eta c}$ such that

$$
\begin{aligned}
\left\|q_{\eta}\right\|_{\infty} \leq & \varsigma_{q \eta e \eta}\left\|e_{\eta}\right\|_{\infty}+\varsigma_{q \eta e \xi}\left\|e_{\xi}\right\|_{\infty}+\varsigma_{q \eta e \eta 2}\left\|e_{\eta}\right\|_{\infty}^{2} \\
& +\varsigma_{q \eta e \xi 2}\left\|e_{\xi}\right\|_{\xi}^{2}+\varsigma_{q \eta e \eta \xi}\left\|e_{\eta}\right\|_{\infty}\left\|e_{\xi}\right\|_{\infty}+\varsigma_{q \eta c} .
\end{aligned}
$$

Furthermore, from (19), (29), and (30), one can have that

$$
\begin{aligned}
\left\|e_{\xi}\right\|_{\infty} \leq & \gamma_{e_{\xi}(0)}+\mu_{\xi} \varsigma_{q \xi e \eta}\left\|e_{\eta}\right\|_{\infty}+\mu_{\xi} \varsigma_{q \xi e \xi}\left\|e_{\xi}\right\|_{\infty} \\
& +\mu_{\xi} \varsigma_{q \xi c}, \\
\left\|e_{\eta}\right\|_{\infty} \leq & \gamma_{e_{\eta}(0)}+\mu_{\eta} \varsigma_{q \eta e \eta}\left\|e_{\eta}\right\|_{\infty}+\mu_{\eta} \varsigma_{q \eta e \xi}\left\|e_{\xi}\right\|_{\infty} \\
& +\mu_{\eta} \varsigma_{q \eta c}+\mu_{\eta} \varsigma_{q \eta e \eta 2}\left\|e_{\eta}\right\|_{\infty}^{2}+\mu_{\eta} \varsigma_{q \eta e \xi 2}\left\|e_{\xi}\right\|_{\xi}^{2} \\
& +\mu_{\eta} \varsigma_{q \eta e \eta \xi}\left\|e_{\eta}\right\|_{\infty}\left\|e_{\xi}\right\|_{\infty} .
\end{aligned}
$$

Combining (30) and (31), one can obtain positive constants $\varsigma_{q \eta e \eta}^{\prime}, \varsigma_{q \eta e \eta 2}^{\prime}$, and $\varsigma_{q \eta c}^{\prime}$ satisfying

$$
\left\|q_{\eta}\right\|_{\infty} \leq \varsigma_{q \eta e \eta}^{\prime}\left\|e_{\eta}\right\|_{\infty}+\varsigma_{q \eta e \eta 2}^{\prime}\left\|e_{\eta}\right\|_{\infty}^{2}+\varsigma_{q \eta c}^{\prime} .
$$

If $\left\|e_{\eta}\right\|_{\infty}$ satisfies that

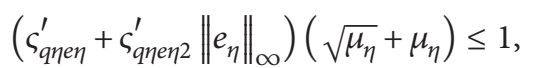

then one can obtain that

$$
\left\|q_{\eta}\right\|_{\infty} \leq \frac{\left(\gamma_{e_{\eta}(0)}+\varsigma_{q \eta}^{\prime} / \varsigma_{q \eta e \eta}^{\prime}\right)}{\sqrt{\mu_{\eta}}}
$$

It follows that

$$
\begin{aligned}
\max _{j}\left|e_{\eta j}(t)\right| \leq \max _{j}\left|c_{\eta j}^{T} e^{A_{\eta H} t} e_{\eta}(0)\right| \\
+\sqrt{\mu_{\eta}}\left(\gamma_{e_{\eta}(0)}+\frac{\varsigma_{q \eta c}^{\prime}}{\varsigma_{q \eta e \eta}^{\prime}}\right) .
\end{aligned}
$$


TABLE 1: Helicopter parameters.

\begin{tabular}{lccccc}
\hline Parameter & Value & Parameter & Value & Parameter & Value \\
\hline$b_{1}^{N}$ & -9.8 & $b_{2}^{N}$ & 9.8 & $b_{3}^{N}$ & 1 \\
$b_{4}^{N}$ & 0.32 & $b_{5}^{N}$ & 0.32 & $b_{6}^{N}$ & 0.8 \\
\hline
\end{tabular}

It should be noted that, from (33), one can obtain the attractive region of the travel channel

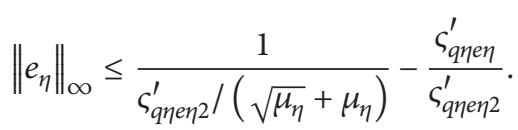

Define $\varsigma_{q \eta}^{\prime}=\gamma_{e_{\eta}(0)}+\varsigma_{q \eta c}^{\prime} / \varsigma_{q \eta e \eta}^{\prime}$. If $e_{\eta}(0)$ is in the attractive region, $e_{\eta}(t)$ can remain inside it if

$$
\gamma_{e_{\eta}(0)}+\sqrt{\mu_{\eta}} \varsigma_{q \eta}^{\prime}<\frac{1}{\varsigma_{q \eta e \eta 2}^{\prime} /\left(\sqrt{\mu_{\eta}}+\mu_{\eta}\right)}-\frac{\varsigma_{q \eta e \eta}^{\prime}}{\varsigma_{q \eta e \eta 2}^{\prime}}
$$

If $\mu_{\eta}$ is sufficiently small, one can see that the above inequality holds. So if the initial state of the angles satisfies that

$$
\left\|e_{\eta}(0)\right\|_{\infty} \leq \gamma_{e_{\eta}(0)}+\sqrt{\mu_{\eta}}\left(\gamma_{e_{\eta}(0)}+\frac{\varsigma_{q \eta c}^{\prime}}{\varsigma_{q \eta e \eta}^{\prime}}\right)
$$

one can obtain (33).

From (19), (29), and (34), one can have that

$$
\left\|q_{\xi}\right\|_{\infty} \leq \frac{\varsigma_{q \xi e \eta} \gamma_{e_{\eta}(0)}+\varsigma_{q \xi e \eta} \sqrt{\mu_{\eta}} \varsigma_{q \eta}^{\prime}+\varsigma_{q \xi e \xi} \gamma_{e_{\xi}(0)}+\varsigma_{q \xi c}}{1-\mu_{\xi} \varsigma_{q \xi e \xi}} .
$$

Moreover, from (18) and (39), one has that

$$
\begin{aligned}
& \max _{j}\left|e_{\xi j}(t)\right| \\
& \leq \max _{j}\left|c_{\xi j}^{T} e^{A_{\xi H} t} e_{\xi}(0)\right| \\
& \quad+\mu_{\xi} \frac{\varsigma_{q \xi e \eta} \gamma_{e_{\eta}(0)}+\varsigma_{q \xi e \eta} \sqrt{\mu_{\eta}} \varsigma_{q \eta}^{\prime}+\varsigma_{q \xi e \xi} \gamma_{e_{\xi}(0)}+\varsigma_{q \xi c}}{1-\mu_{\xi} \varsigma_{q \xi e \xi}}
\end{aligned}
$$

If $\mu_{\xi}$ and $\mu_{\eta}$ are sufficiently small, then from (35) and (40), one can get positive constants $T_{\xi}$ and $T_{\eta}$ such that (28) holds.

\section{Simulation Results}

The reference signals of the four channels are generated by $r_{i}(p)=r_{i}^{i c}(p) /\left(\tau_{r i} p+1\right)^{2}(i=x, y, z, \psi)$, respectively, where $r_{i}^{i c}(p)(i=x, y, z, \psi)$ are the reference input commands. Set $\tau_{r x}=0.7, \tau_{r y}=0.7, \tau_{r z}=0.7$, and $\tau_{r \psi}=0.7$. The helicopter parameters are shown in Table 1 , followed by the controller parameters in Table 2.

The helicopter is required to carry out aggressive missions in harsh conditions; that is, amplitudes for the references $r_{x}, r_{y}, r_{z}$, and $r_{\psi}$ are $10 \mathrm{~m}, 10 \mathrm{~m}, 10 \mathrm{~m}$, and $60 \mathrm{deg}$, respectively; all of the parametric uncertainties are assumed up to $40 \%$ of the nominal values, and the rotorcraft is subject to persistent external disturbances $d_{1}=0.2, d_{2}=0.2, d_{3}=3, d_{4}=0.2$,

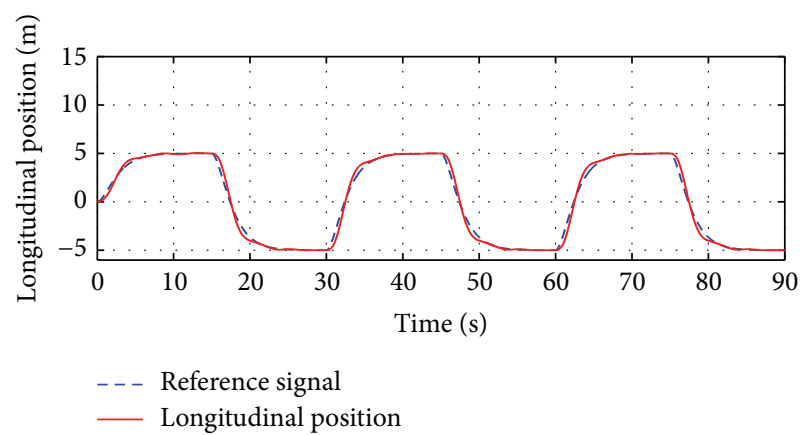

(a) Longitudinal response

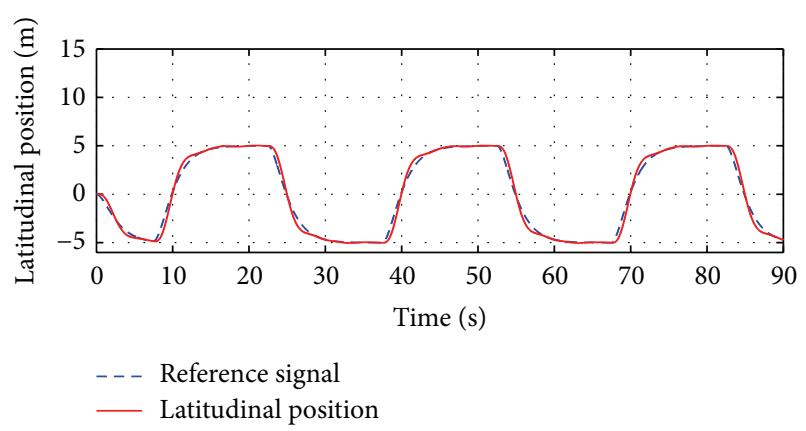

(b) Latitudinal response

FIGURE 2: Responses of the longitudinal position and the latitudinal position with RCT.

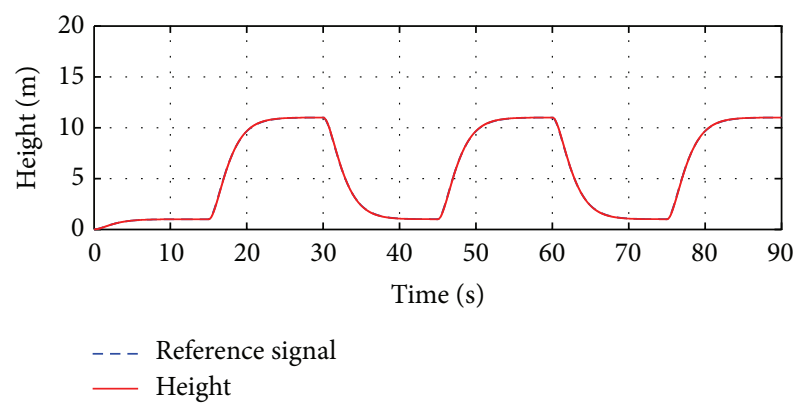

(a) Height response

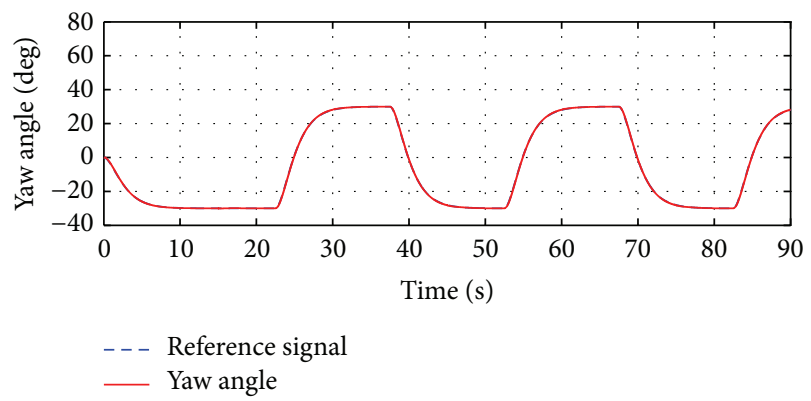

(b) Yaw response

FIGURE 3: Responses of the height and the yaw angle with RCT.

$d_{5}=0.2$, and $d_{6}=1$. The external disturbance $d_{3}$ is larger than other disturbances because the aerial vehicle is likely subject to the ground effect when it flies near the ground.

Figures 2 and 3 show the simulation results of the trajectory tracking of the desired references. The rotorcraft 
TABLE 2: Controller parameters.

\begin{tabular}{lccccccc}
\hline Parameter & Value & Parameter & Value & Parameter & Value & Parameter & Value \\
\hline$k_{\xi 1}^{p}$ & 2 & $k_{\xi 1}^{d}$ & 1 & $f_{\xi 1}$ & 100 & $g_{\xi 1}$ & 20 \\
$k_{\xi 2}^{p}$ & 2 & $k_{\xi 2}^{d}$ & 1 & $f_{\xi 2}$ & 100 & $g_{\xi 2}$ & 20 \\
$k_{\xi 3}^{p}$ & 10 & $k_{\xi 3}^{d}$ & 25 & $f_{\xi 3}$ & 100 & $g_{\xi 3}$ & 20 \\
$k_{\eta 1}^{p}$ & 20 & $k_{\eta 1}^{d}$ & 100 & $f_{\eta 1}$ & 2500 & $g_{\eta 1}$ & 500 \\
$k_{\eta 2}^{p}$ & 20 & $k_{\eta 2}^{d}$ & 100 & $f_{\eta 2}$ & 2500 & $g_{\eta 2}$ & 500 \\
$k_{\eta 3}^{p}$ & 10 & $k_{\eta 3}^{d}$ & 25 & $f_{\eta 3}$ & 100 & $g_{\eta 3}$ & 20 \\
\hline
\end{tabular}

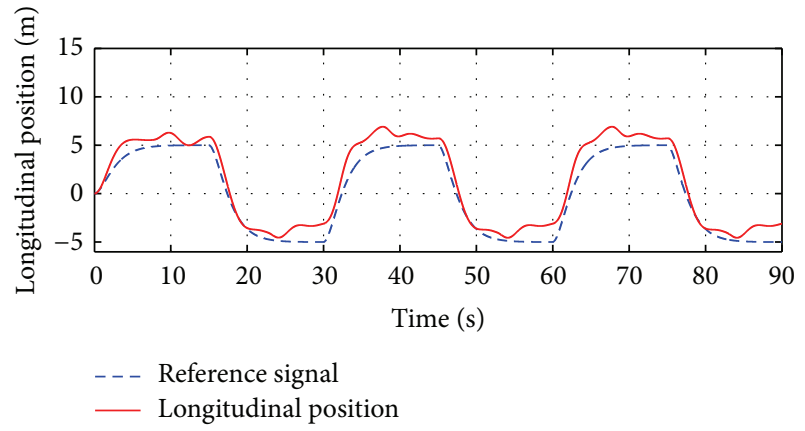

(a) Longitudinal response

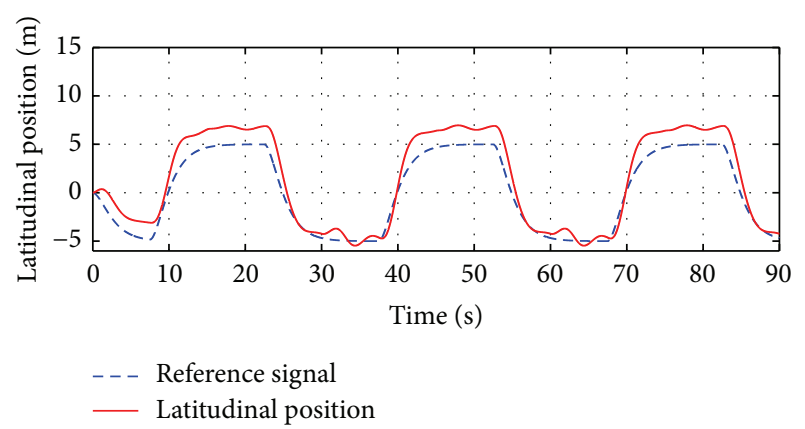

(b) Latitudinal response

FIGURE 4: Responses of the longitudinal position and the latitudinal position without RCT.

starts from $x(0)=0, y(0)=0, z(0)=0$, and $\psi(0)=0$. $x, y, z$, and $\psi$ are required to follow large references $r_{x}, r_{y}, r_{z}$, and $r_{\psi}$ simultaneously. Besides, from Model (2), it can be seen that there exist inter-axis couplings between channels. By the proposed robust control method, good tracking performance can be achieved for aggressive maneuvers under the influence of various uncertainties. Furthermore, the proposed robust controller is compared with the nominal controller. The corresponding responses of $x, y, z$, and $\psi$ without the RCT (robust compensating technique) are shown in Figures 4 and 5. From these figures, it can be seen that the proposed control method achieves better dynamical and steady-state performance especially in the longitudinal and latitudinal directions. The tracking performance of the height and yaw angle is only improved slightly, because the nonlinear dynamical models of these two channels are simple compared to the models in the longitudinal and latitudinal channels as shown in the nonlinear model (2). Because the quadrotor is

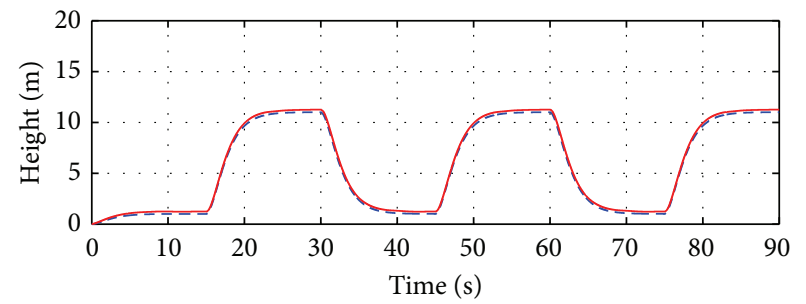

- - - Reference signal

(a) Height response

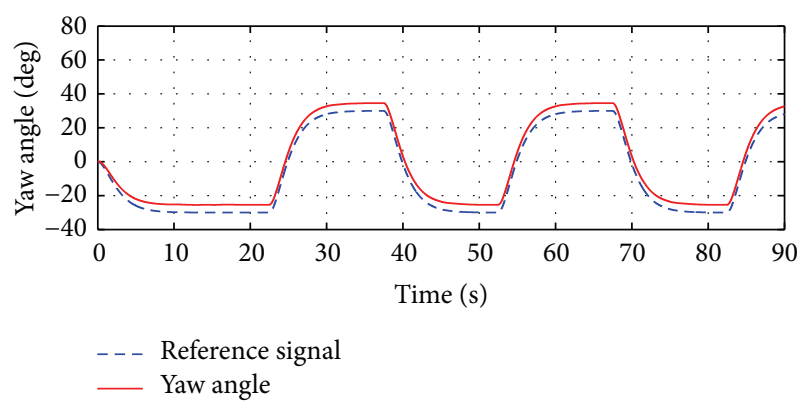

(b) Yaw response

FIGURE 5: Responses of the height and the yaw angle without RCT.

underactuated in the longitudinal and latitudinal directions, the pitch and roll angles are required to track the desired references obtained by the longitudinal and latitudinal position tracking errors. The responses of the two angles are shown in Figure 6. Good dynamical and steady-state tracking performance is also achieved for the pitch and roll angles.

\section{Conclusions}

A robust controller was proposed to achieve the motion control for a hexarotor system. The proposed control structure is a hierarchical scheme that consists of two control loops: an attitude loop and a position loop. Both the attitude and position control loops include a nominal controller to achieve the desired tracking performances and a robust compensator to restrain the influences of uncertainties such as nonlinear dynamics, coupling, parametric uncertainties, and external disturbances. It is proven that the tracking errors are ultimately bounded and the boundaries can be specified. 


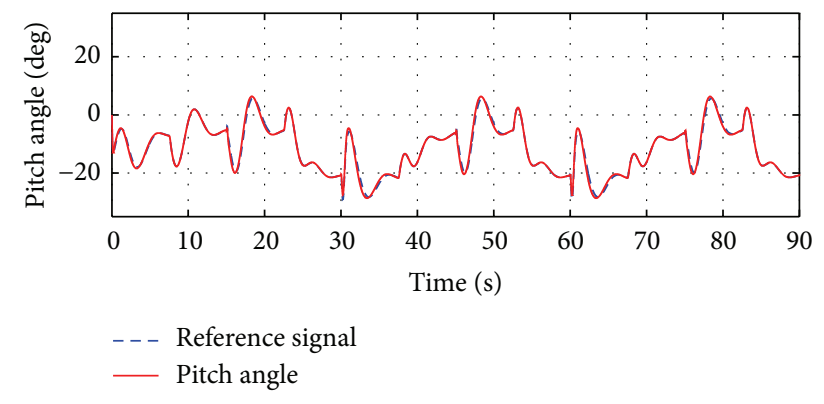

(a) Pitch response

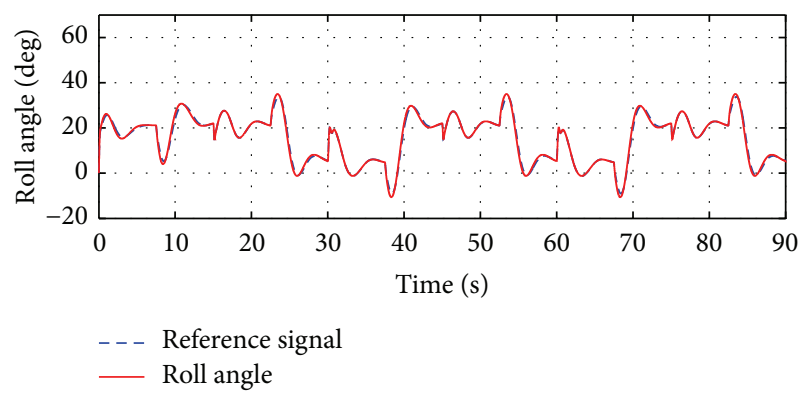

(b) Roll response

FIGURE 6: Responses of the pitch angle and the roll angle with RCT.

Simulation results on the hexarotor helicopter demonstrate the effectiveness of the proposed control method.

\section{Conflict of Interests}

The authors declare that there is no conflict of interests regarding the publication of this paper.

\section{Acknowledgments}

This work was supported by the National Natural Science Foundation of China under Grants nos. 61374054 and 61473324 and the Fundamental Research Funds for the Central Universities under Grants nos. YWF-14-RSC-036 and YWF-14-YHXY-019.

\section{References}

[1] E. Prempain and I. Postlethwaite, "Static $H_{\infty}$ loop shaping control of a fly-by-wire helicopter," Automatica, vol. 41, no. 9, pp. 1517-1528, 2005.

[2] K. M. Peng, G. W. Cai, B. M. Chen, M. Dong, K. Y. Lum, and T. H. Lee, "Design and implementation of an autonomous flight control law for a UAV helicopter," Automatica, vol. 45, no. 10, pp. 2333-2338, 2009.

[3] L. Marconi and R. Naldi, "Robust full degree-of-freedom tracking control of a helicopter," Automatica, vol. 43, no. 11, pp. 1909-1920, 2007.

[4] I. A. Raptis, K. P. Valavanis, and W. A. Moreno, "A novel nonlinear backstepping controller design for helicopters using the rotation matrix," IEEE Transactions on Control Systems Technology, vol. 19, no. 2, pp. 465-473, 2011.
[5] Q. Qu, F. Chen, B. Jiang, and G. Tao, "Integral sliding mode control for helicopter via disturbance observer and quantum information technique," Mathematical Problems in Engineering, vol. 2015, Article ID 938246, 7 pages, 2015.

[6] G. M. Hoffmann, H. Huang, S. L. Waslander, and C. J. Tomlin, "Precision flight control for a multi-vehicle quadrotor helicopter testbed," Control Engineering Practice, vol. 19, no. 9, pp. 1023-1036, 2011.

[7] G. V. Raffo, M. G. Ortega, and F. R. Rubio, "An integral predictive/nonlinear $H_{\infty}$ control structure for a quadrotor helicopter," Automatica, vol. 46, no. 1, pp. 29-39, 2010.

[8] K. Alexis, G. Nikolakopoulos, and A. Tzes, "Switching model predictive attitude control for a quadrotor helicopter subject to atmospheric disturbances," Control Engineering Practice, vol. 19, no. 10, pp. 1195-1207, 2011.

[9] P. Castillo, A. Dzul, and R. Lozano, "Real-time stabilization and tracking of a four-rotor mini rotorcraft," IEEE Transactions on Control Systems Technology, vol. 12, no. 4, pp. 510-516, 2004.

[10] A. Tayebi and S. McGilvray, "Attitude stabilization of a VTOL quadrotor aircraft," IEEE Transactions on Control Systems Technology, vol. 14, no. 3, pp. 562-571, 2006.

[11] S. Bertrand, N. Guénard, T. Hamel, H. Piet-Lahanier, and L. Eck, "A hierarchical controller for miniature VTOL UAVs: design and stability analysis using singular perturbation theory," Control Engineering Practice, vol. 19, no. 10, pp. 1099-1108, 2011.

[12] T. Hamel and R. Mahony, "Visual servoing of an under-actuated dynamic rigid-body system: an image-based approach," IEEE Transactions on Robotics and Automation, vol. 18, no. 2, pp. 187198, 2002.

[13] A. Das, K. Subbarao, and F. Lewis, "Dynamic inversion with zero-dynamics stabilisation for quadrotor control," IET Control Theory and Applications, vol. 3, no. 3, pp. 303-314, 2009.

[14] J. F. Guerrero-Castellanos, N. Marchand, A. Hably, S. Lesecq, and J. Delamare, "Bounded attitude control of rigid bodies: realtime experimentation to a quadrotor mini-helicopter," Control Engineering Practice, vol. 19, no. 8, pp. 790-797, 2011.

[15] Z. Zuo, "Trajectory tracking control design with commandfiltered compensation for a quadrotor," IET Control Theory \& Applications, vol. 4, no. 11, pp. 2343-2355, 2010.

[16] I. A. Raptis, K. P. Valavanis, and W. A. Moreno, "A novel nonlinear backstepping controller design for helicopters using the rotation matrix," IEEE Transactions on Control Systems Technology, vol. 19, no. 2, pp. 465-473, 2011.

[17] Y.-S. Zhong, "Robust output tracking control of SISO plants with multiple operating points and with parametric and unstructured uncertainties," International Journal of Control, vol. 75, no. 4, pp. 219-241, 2002.

[18] H. Liu, Y. Bai, G. Lu, and Y. Zhong, "Robust attitude control of uncertain quadrotors," IET Control Theory and Applications, vol. 7, no. 11, pp. 1583-1589, 2013.

[19] H. Liu, X. Wang, and Y. Zhong, "Quaternion-based robust attitude control for uncertain robotic quadrotors," IEEE Transactions on Industrial Informatics, vol. 11, no. 2, pp. 406-415, 2015.

[20] H. Liu, D. Li, J. Xi, and Y. Zhong, "Robust attitude controller design for miniature quadrotors," International Journal of Robust and Nonlinear Control, 2015.

[21] H. Liu, G. Lu, and Y. Zhong, "Robust LQR attitude control of a 3-DOF laboratory helicopter for aggressive maneuvers," IEEE Transactions on Industrial Electronics, vol. 60, no. 10, pp. 46274636, 2013. 


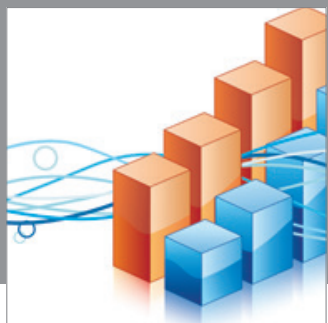

Advances in

Operations Research

mansans

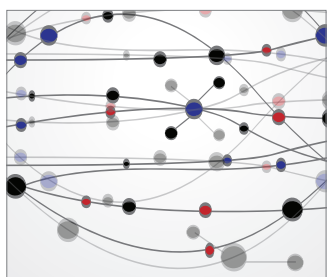

The Scientific World Journal
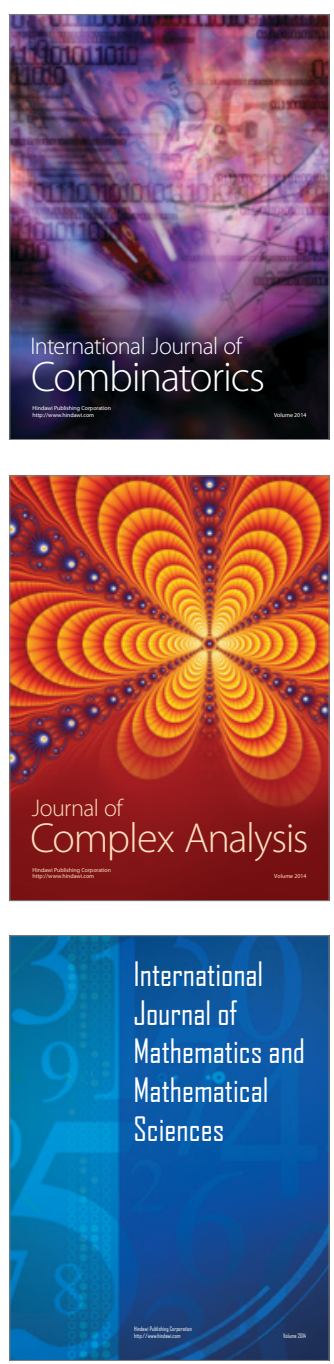
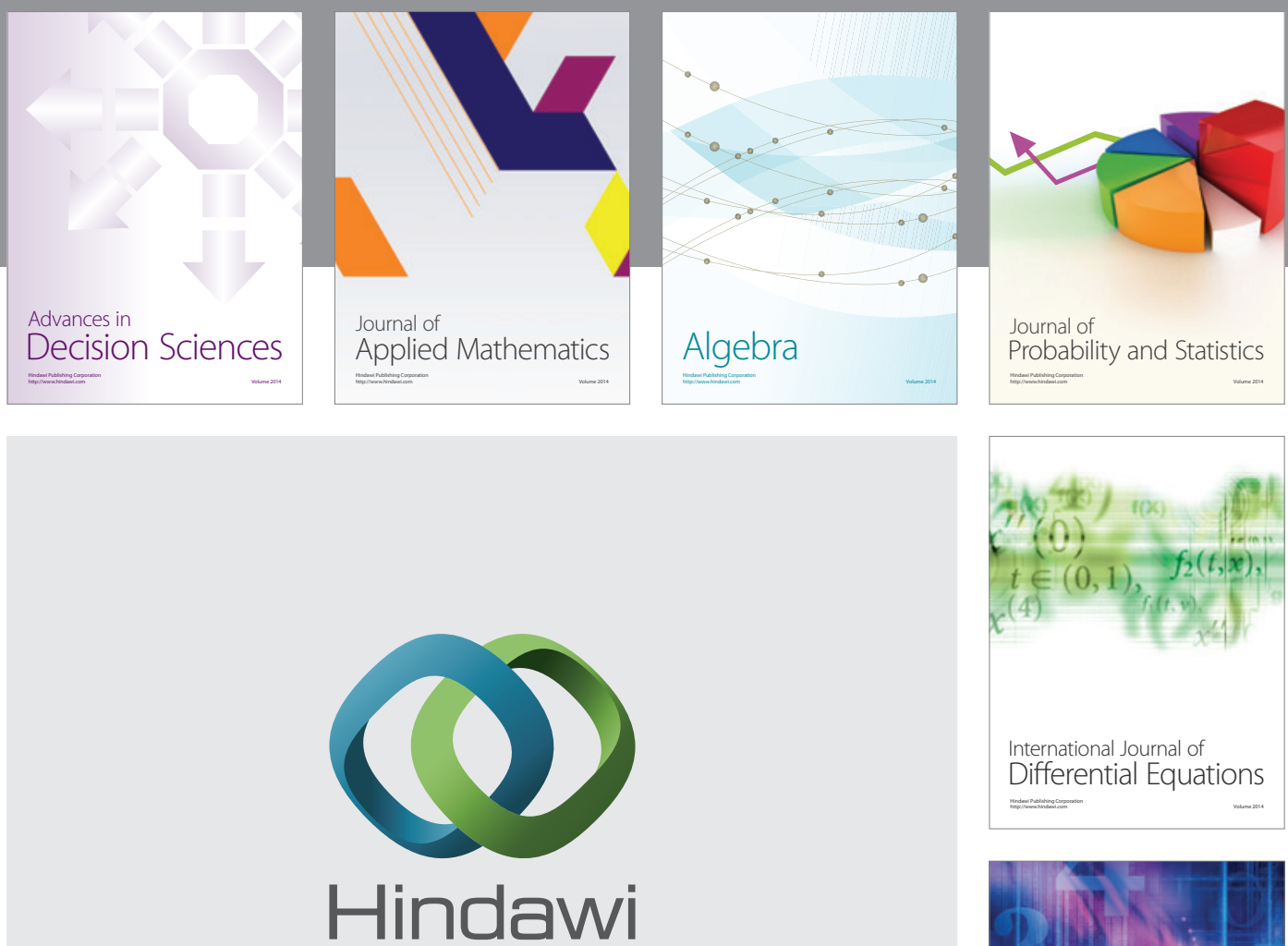

Submit your manuscripts at http://www.hindawi.com
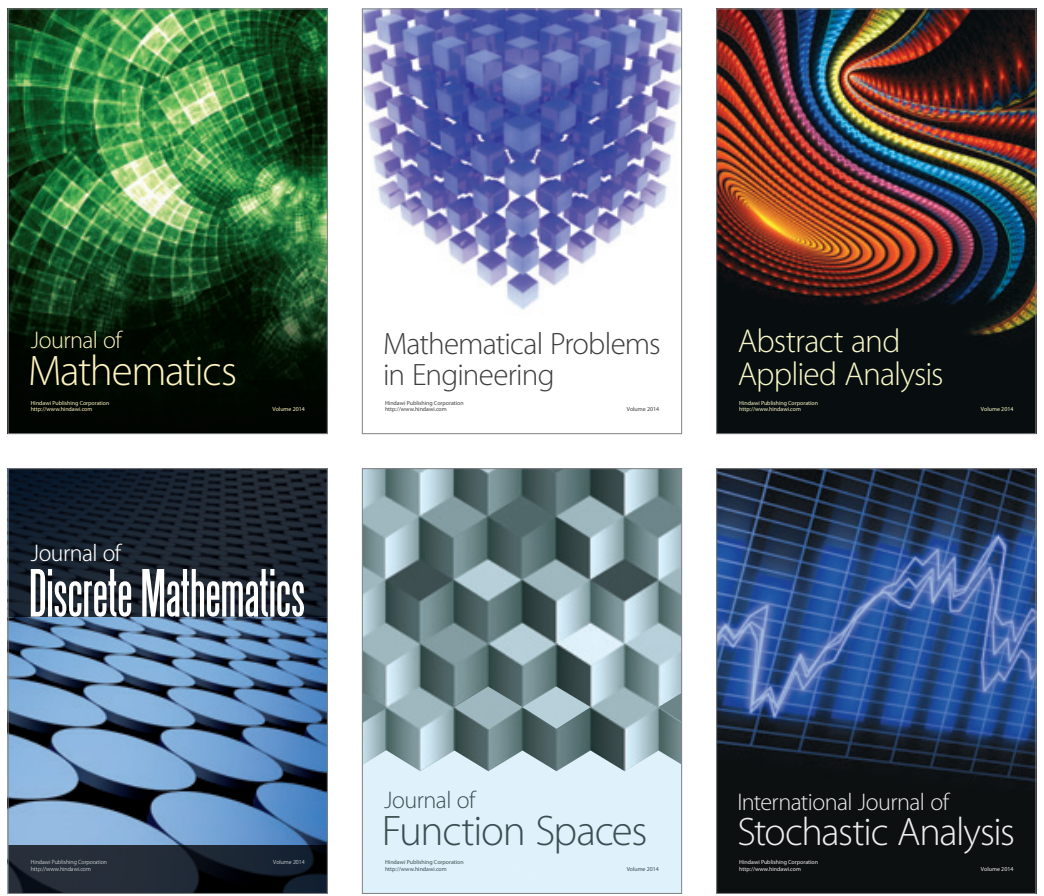

Journal of

Function Spaces

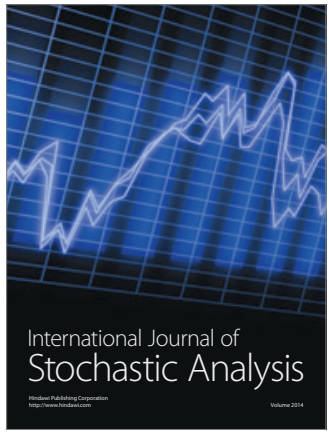

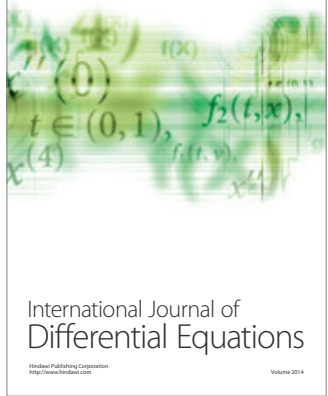
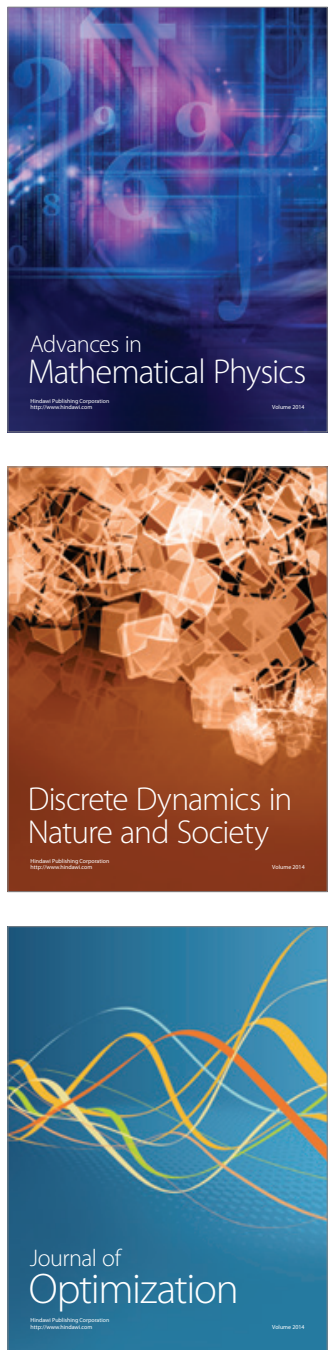